\title{
Una propuesta de clase con GeoGebra: el dominio, rango y la transformación de funciones construyendo animaciones ${ }^{1}$
}

\author{
A proposed class with GeoGebra: domain, range and the \\ transformation of functions built animations
}

\section{A classe proposto com GeoGebra: domínio, alcance e da transformação das funções animações construídas}

Recibido: mayo de 2013

Aceptado: agosto de 2013
Ricardo Rey ${ }^{2}$

Gilberto Serrano ${ }^{3}$

Sandra Rojas ${ }^{4}$

William Jiménez ${ }^{5}$

\section{Resumen}

Este documento presenta algunos resultados obtenidos en estudiantes para profesor de educación básica con énfasis en matemáticas, en el curso de Didáctica de la Geometría, guiados por el estudio de movimientos rígidos como translaciones y reflexiones, y transformaciones de compresión en representaciones gráficas de un sistema de coordenadas cartesiano en un plano real, haciendo énfasis en el estudio de los comportamientos del dominio y rango de las funciones. La propuesta se desarrolla dentro de la aplicación de una actividad cuyo propósito es hacer uso del software de geometría dinámica "GeoGebra" y sus herramientas, para construir animaciones visualmente atractivas, donde se hiciera evidente el manejo de las transformaciones mencionadas, pretendiendo una reflexión sobre su comportamiento.

Palabras clave: classroom, teaching resources, computing resources, software, functions, transformation of functions.

\section{Abstract}

This paper presents some results from student teachers of basic education with an emphasis in mathematics, in the course of Teaching Geometry, guided by the study of rigid motions such as translations and reflections, and compression transformations graphical representations of a system Cartesian coordinates in a real plane, with emphasis on the study of the

Articulo de Investigación

2 Universidad Distrital Francisco Jose de Caldas, Bogotá, Colombia. Contacto: lordking_28@hotmail.com

3 Universidad Distrital Francisco Jose de Caldas, Bogotá, Colombia. Contacto: juniorsq@gmail.com

4 Instituto Pedagógico Nacional de Colombia, Colombia. Contacto: rojastolosa@yahoo.com.ar

5 Universidad Manuela Beltran, Bogotá, Colombia. Contacto: williamajg@hotmail.com 
behavior of the domain and range of functions. The proposal is developed within the implementation of an activity whose purpose is to make use of dynamic geometry software "GeoGebra" and its tools to build visually appealing animations where management became apparent transformations mentioned, trying to reflect on their behavior.

Keywords: classroom, teaching resources, computing resources, software, functions, transformation of functions.

\section{Resumo}

Este artigo apresenta alguns resultados de futuros professores da educação básica com ênfase em matemática, no curso de ensino da geometria, guiada pelo estudo dos movimentos rígidos, como traduções e reflexões e transformações de compressão representações gráficas de um sistema coordenadas cartesianas em um plano real, com ênfase sobre o estudo do comportamento do domínio e gama de funções. A proposta é desenvolvida no âmbito da implementação de uma atividade cujo objetivo é fazer com que o uso de software de geometria dinâmica "GeoGebra" e suas ferramentas para criar animações visualmente atraente onde a gestão tornou-se transformações aparentes mencionado, tentando refletir sobre a sua comportamento.

Palavras-chave: sala de aula, recursos pedagógicos, recursos de computação, software, funções de transformação de funções.

\section{Presentación del problema}

En el año 2011 en la cátedra de Didáctica de la Geometría de quinto semestre del proyecto curricular de Licenciatura en Educación Básica con Énfasis en Matemáticas en la Universidad Distrital Francisco José de Caldas (Bogotá, Colombia), se proponen actividades enmarcadas en la resolución de problemas haciendo énfasis en el uso de las tecnologías en el aula como: software matemáticos e instrumentos tecnológicos para la enseñanza de las matemáticas. Una de dichas actividades se centró en el tema de "transformaciones de funciones en el plano real" haciendo uso del software de geometría dinámica "GeoGebra". Se propuso una situación basada en la elaboración de animaciones creativas donde la representación gráfica en un sistema de coordenadas cartesiano de funciones, sus dominios y rangos, movimientos rígidos como translaciones, reflexiones, y transformaciones de compresión, fueron los elementos básicos para la construcción de la animación.
Para abordar la propuesta de clase se hacen necesarios algunos conceptos teóricos que abordaremos más adelante; sin embargo, es importante mencionar que la propuesta no tiene como objetivo la enseñanza de los conceptos sino su manipulación y el reconocimiento de las características principales básicas para la creación de una animación visualmente atractiva.

\section{Marco teórico}

En nuestros precedentes teóricos consideraremos algunos conceptos básicos como la función, su dominio y su rango, además de las trasformaciones de una función vistas como movimientos rígidos. Dichos conceptos y procesos son los que se pretenden afianzar en la actividad propuesta mediante el uso de GeoGebra.

Llamaremos función de un conjunto en un conjunto, a toda relación que cumpla la condición: 
Para todo, existe y sólo uno / .

Llamaremos dominio y rango de la función $\mathrm{f}$ a los conjuntos D(f) e I(f) de acuerdo con Muños (2002).

Para nuestro caso particular, funciones en los reales (tendremos que, en este caso es usual presentar la función con la notación $\mathrm{y}=\mathrm{f}(\mathrm{x})$.
Jiménez et al. (2011) afirman que las funciones reales pueden originar otras a través de trasformaciones de éstas haciendo operaciones aditivas o multiplicativas, los resultados al realizar estas se resumen en el esquema 1

Esquema 1. Resumen de las características de la transformación de funciones

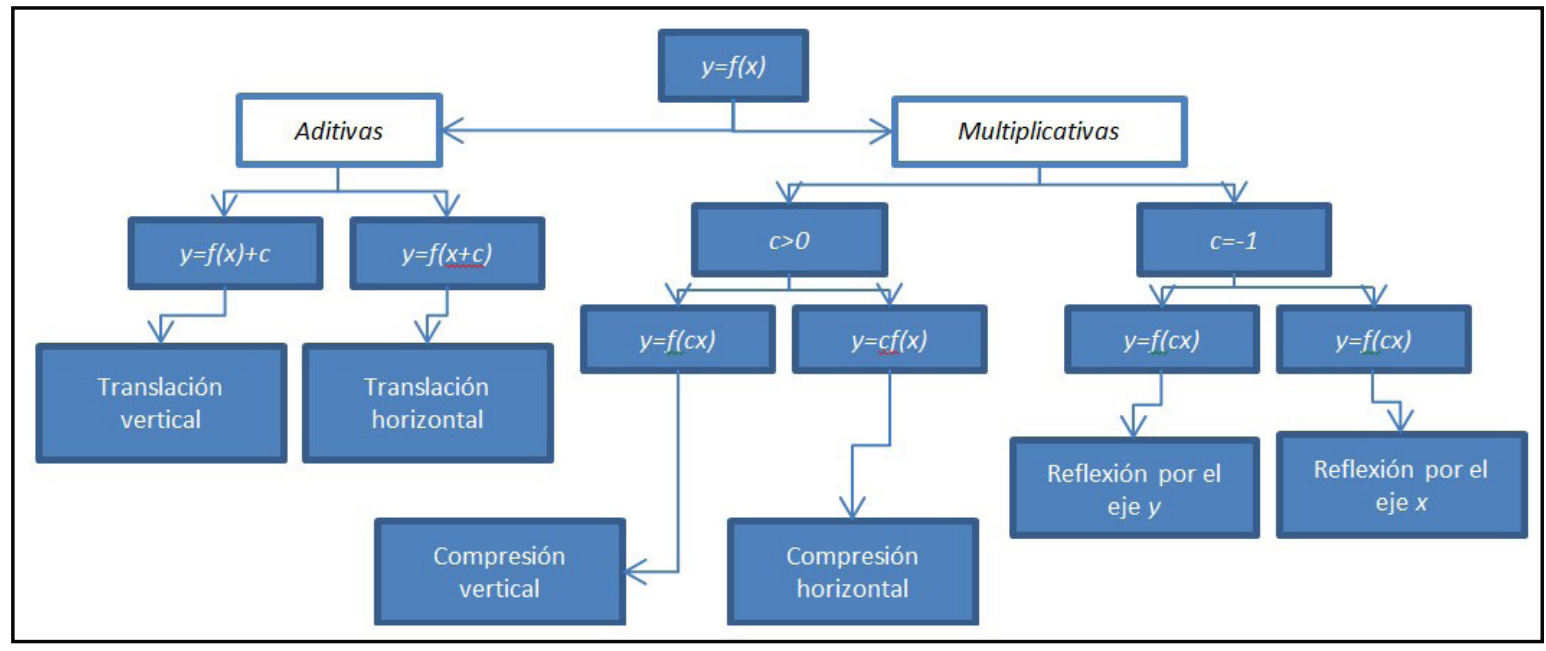

Fuente: Elaboración propia

\section{Consolidación}

Lo primero es la aplicación de una de las herramientas de GeoGebra. La primera de ellas es el deslizador, el cual se empleó para dar movimiento a las funciones, hacer variar un objeto con un cambio definido en intervalos de números racionales; es decir, se aplica el deslizador para comenzar a animar las representaciones gráficas de las funciones descritas anteriormente.

La actividad da inicio con la explicación de las diversas formas de graficar una función en GeoGebra; la primera de estas es el de la herramienta sin restricción o condicional que sirven para graficar una función con dominio sin poner condición alguna puesto que el software mismo identifica el dominio de la función.

La segunda forma para graficar una función es la herramienta condicional, que sirve para definir funciones a trozos; por ejemplo, para graficar funciones relacionadas con el valor absoluto que dependen de
Figura 1

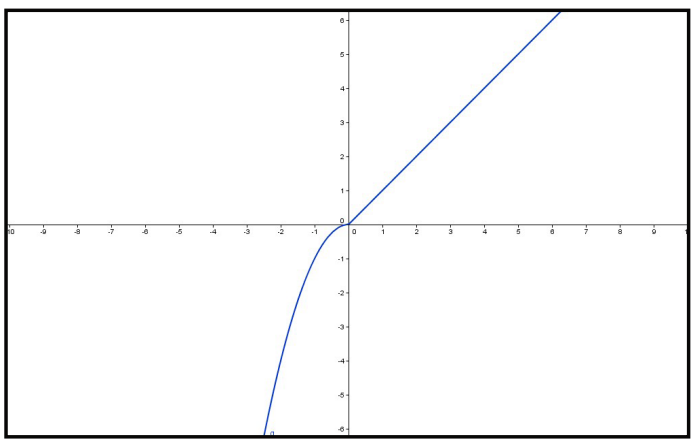

Fuente: Elaboración propia

un intervalo para trazar el segmento de alguna función, se condiciona el dominio. Por ejemplo para la función

Y por último, la tercera forma: función con restricción, ésta sirve para restringir la función en un intervalo dado, donde se restringe el dominio para obtener solamente un trozo de la función (figura 2).

Haciendo uso de éstas formas y de diferentes tipos de funciones, se procede a realizar la construcción de varias imágenes interesantes. Un ejemplo la 
Figura 2

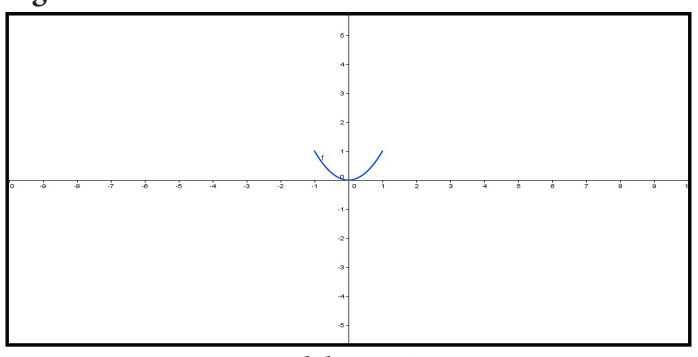

Fuente: Elaboración propia

construcción de una "barca" que está sobre el agua, que se desplaza sobre el mar (figura 3), actividad que se propuso para que los estudiantes la replicaran. Cuando se les pide a ellos que grafiquen de nuevo la barca empiezan a aparecer los errores en las animaciones.

Figura 3

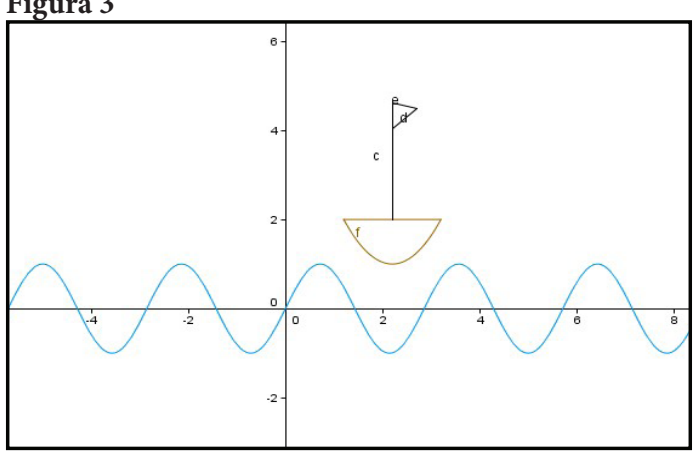

Fuente: Elaboración propia

Figura 4

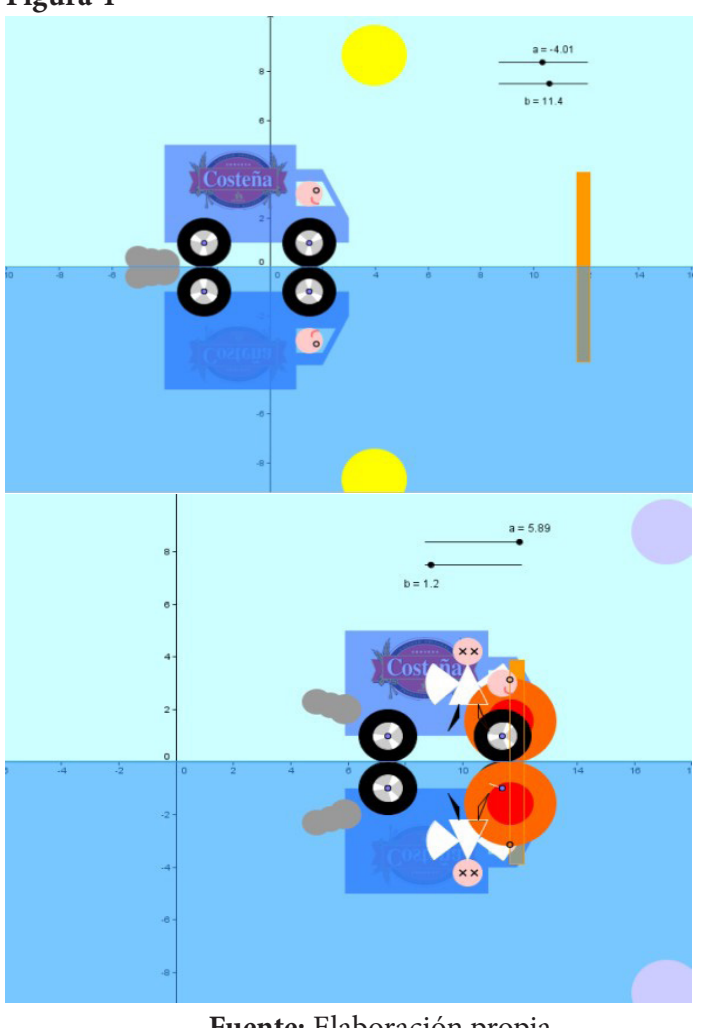

Finalmente se propone a los estudiantes que realicen su propia animación. Algunos ejemplos de las construcciones realizadas se muestran en la figura 4 .

\section{Conclusiones}

El tipo de actividad propuesta exige de parte de los estudiantes para profesor de matemáticas comprender los conceptos que se emplean en las animaciones como dominio, rango, transformaciones de funciones, entre otros, logrando establecer relaciones entre los diferentes sistemas de representación, en este caso, entre el algebraico y el gráfico.

Analizar los errores cometidos en las construcciones permite que los estudiantes reconozcan cuáles son los tipos de errores y dificultades que se presentan en este tipo de actividades e identificar cuáles de ellas son inherentes al concepto mismo y cuales son generadas por el uso del software.

Finalmente, el proponer a los estudiantes para profesor este tipo de actividades les posibilita reconocer otro tipo de enfoque para la enseñanza de las matemáticas escolares, en el que la incentivación y el uso de software matemático y situaciones problemáticas dan sentido a la actividad matemática en el aula.

\section{Referencias}

Jiménez W., Mayorga L., Ahumada J., \& Cuchigay A. (2011). Transformaciones de funciones en coordenadas polares. En memorias del X Encuentro Nacional de Educación Matemáticas y Estadística. Universidad Pedagógica y Tecnológica de Tunja. Tomado de http://www.uptc.edu.co/eventos/2011/ enemes/programacion/documentos/memorias_2011.pdf

Muñoz, Q. (2002). Introducción a la teoría de conjuntos. Ed. Universidad Nacional de Colombia. 